Series A

I. MATHEMATICA

$336 / 9$

\title{
POSITIVE REAL RESOLVENTS AND LINEAR PASSIVE HILBERT SYSTEMS
}

BY

C. L. DOLPH 
Communicated 8 February 1963 by P. J. Myrberg and Olli Lehto 


\title{
Positive real resolvents and linear passive Hilbert systems*
}

\author{
Dedicated to R. S. Phillips on his 50th birthday
}

\section{Introduction}

The theory of dissipative operators in Hilbert space has been extensively developed and applied to a wide variety of problems within recent years. While my earlier prediction of [9] that this theory would soon become a standard part of an advanced mathematical education has not yet been fulfilled, recent advances make it most likely that this soon will be the case. Rather than attempting a complete survey of this rapidly expanding theory, attention here will be mainly focused on a reinterpretation, believed to be new at least in this generality, of a large part of the existing theory from the view-point of the so-called »black box» concept familiar in the mathematics of system engineering. The motivation which led to the point of view to be presented here stems from a question raised privately by R. S. Phillips concerning the reasons why Penzlin and myself were interested in having a Stieltjes type representation for the resolvents of dissipative operators in a half-plane (cf. Dolph and Penzlin [10], and Dolph [9]). As a result of the fact that Phillips in this same conversation also suggested a way of obtaining a stronger result than those referred to; namely, theorem 4.14 of this paper, it is now possible not only to provide an answer to his question which has intrinsic interest in operator theory but also one which points the way toward a theory of the transient behavior of a class of linear systems which can be associated with this representation in a natural way. Since many of the important physical problems of interest today fall into this class, it is hoped that the discussion here will be provocative and will eventually lead to the development of a systematic theory of the transient behavior of continuous linear systems.

To be more specific, the correspondence which exists between the so-called class of positive real functions (admittance or impedances of linear passive networks furnish the best known examples) and the resolvents of maximal dissipative closed operators in the sense of Phillips [70] (for

\footnotetext{
* Part of this research has been supported by a National Science Foundation Grant.
} 
precise definition see $\S 4$ ) will be examined in detail. Interpreting causality to mean that a linear system cannot show a response before a signal is applied, the analytical properties possessed by this class of resolvents makes it possible to generalize the relationship which exists for positive real functions and linear passive systems in the sense of König and Meixner [44], Wu [109], and Youla, Castriota and Carlin [111]. That is, it will be shown that to each resolvent of the subclass of maximal dissipative operators, which are regularly dissipative in the sense of Kato [39], there exists an abstract linear system which is defined in a Hilbert space and which possesses the additional properties of being invariant under time translations, causal, passive (and hence dissipative) and conversely, that the abstract equation of evolution generated by a time invariant regular dissipative operator has a resolvent possessing properties analogous to those of positive real functions. In fact the larger class of resolvents of maximal dissipative closed operators have properties analogous to positive real functions and will be termed the class of "positive real resolvents". Whenever the associated abstract equation of evolution admits a strict solution, it will correspondingly be termed a »linear passive Hilbert system». For precise definition see $\S 4$ and $\S 5$ respectively. In the discussion leading to these results, a new characterization of positive real resolvents will be derived. This characterization is of independent mathematical interest since it is a partial generalization of the relationship which exists between a self-adjoint operator and its resolvent.

The first modern extensive general theory of linear passive systems is due to König and Meixner [44] and was the direct result of Meixner's [58] deduction of the condition of passivity from thermodynamic considerations. This theory emphasizes continuity properties and does not use the results of Hilbert space theory for functions in contrast to that of $\mathrm{Wu}$ [109], Youla et al [111]. The last named authors, however, made an important addition to the general structure of these theories since they were in fact able to show that Meixner's condition of passivity was sufficient to guarantee causality. Their considerations will be extended to the more general case treated here in $\$ 5$.

In addition to these treatments which, with the exception of work of König [43], have all been concerned with the scalar Hilbert space theory, Fourès and Segal [16] discussed causality for an abstract linear system within a framework closely related to that which will be treated here. They considered a class of inputs and outputs consisting of the pre-Hilbert spaces of linear sets of square integrable functions on a real vector group to a countable complex Hilbert space $K$. Under reasonable condition they were able to establish the existence of a "gain function» in their terminology - a resolvent in ours - such that in terms of the Fourier transform on the 
dual group, the transformed output was related to the transformed input by simple multiplication. Moreover, for a bounded set of such operators, invariant under translation, they were able to prove that causality with respect to a cone was equivalent to the existence of a bounded analytic extension of the "gain» to the "tube» over the dual of the group. An interesting application of their results in combination with the theory of the shift operator due originally to Beurling [3] and then generalized by Lax [47] and Halmos [25] has just been made by Lax and Phillips [50], [51].

Here the group will be specialized to the real line and the integral over the group to the Bochner integral. Also semi-group rather than Fourier transform methods will be used and in fact it is the analogy which exists between Laplace transform theory and semi-group theory (cf. Hille and Phillips [29], and Phillips [71]) which has been the main guide leading to the theory of positive real resolvents which will be presented here. Within this framework, the fact that the resolvent has an analytic extension to a whole half-plane follows readily from known theorems of Sz.-Nagy, Phillips and Stone - see theorem 4.14. While the author believes that many of the new results presented here unquestionably have their counterparts in the Fourès-Segal theory he does not feel competent to so phrase and prove them within the more general group context.

The results so far achieved indicate clearly that the analogy between Laplace transform theory and semi-group theory is capable of much further exploitation, and it is our firm belief that it contains the seeds of a new mathematical discipline - the theory of the transient behavior of continuous linear systems just as the theory of Laplace transformations has given rise to the theory of the transient behavior of discrete linear systems.

Some of the general features and advantages of such a theory are already apparent from the known examples discussed by Titchmarsh [97], [98], Friedman [18], Dolph [9], Beck and Nussenzveig [2], Nussenzveig [62], [63], and Petzold [68]. In all of these cases it has been possible to continue the matrix elements formed with the resolvent and elements of compact support from the original half-plane of analyticity into at least part of the other half-plane where new complex singularities are then found. These new singularities, while not part of the spectrum, give rise to the analogue of the Heaviside expansion theorem for the associated continuous system. The resulting series expansion is in terms of transient, non-orthogonal modes, which have no individual existence but which do exist collectively and yield a representation which appears to have far wider and superior convergent properties than any other that are known (cf. for example, the discussion in [63]). Loosely speaking this representation can be said to represent the "peaks» or "resonances» in the continuous spectrum of the operator and as already recognized by Heitler [28] - their use facilitates 
many of the calculations in physics where detailed consideration of the continuous spectra is necessary. In addition, these examples already make it clear, because of exponential growth associated with the complex singularities, that it is only matrix elements of the above form that offer any possibility for continuation within the framework of a Hilbert space theory. Thus one must conclude with Höhler [30] that attempts by Schönberg $[79]-[81]$ to perform the corresponding continuation of the resolvent are incorrect as stated.

This does not, of course, preclude the possibility that the use of a different space might result in a simpler, conceptually more satisfying theory. A promising beginning in this direction has apparently been made by A. Grossman of the Institute for Advanced Study ${ }^{1}$. While no details are yet available, he appears to have been able to imbed Hilbert spaces in a distribution space large enough to admit functions of exponential growth and still small enough so that Schrödinger's equation still has a unique solution. Moreover, in this space the resolvent itself can be continued analytically, and its complex singularities appear naturally on Riemann surfaces as in the spherical shell model treated in detail by Petzold [68] and Nussenzveig [63]. The details should prove most interesting and their publication is eagerly awaited.

Returning to the Hilbert space situation, little if anything is known in general concerning the possibility of continuing the matrix elements of the resolvent of a general maximal dissipative closed operator or even of a self-adjoint operator. Ladyženskaja [45] succeeded in carrying out a continuation for the scattering integral equation associated with a wave equation having a potential of compact support but the resulting continuation was too limited to lead to new singularities. For the potential free wave equation in three dimensions Lax and Phillips [50], [51] have recently succeeded in analytically continuing the associated scattering matrix from one half-plane into a strip in the other halfplane with important consequences for the decay problem. These consequences are sketched in Lax, Morawetz and Phillips [49].

Some information concerning when such continuation is not useful has recently been deduced by McKelvey [5 $]$. For the class of minimal selfadjoint extensions of symmetric operators, he was able to prove that the representation of the resolvent of a symmetric operator in the form of the projection of a resolvent of its self-adjoint extension in a half-plane retained its meaning on an interval of the axis if and only if the resolvent of the self-adjoint extension could be analytically continued through this interval and that this, in turn, required it to be continuous and self-adjoint on the

1 Personal conversations with Dr. A. Grossman and Professor B. Zumino (cf. also the note added in proof-reference, p. 33). 
interval. These results imply that the corresponding matrix elements would be analytic across such an interval and thus could not introduce the complex singularities necessary for the transient theory.

Perhaps the most promising attack on the continuation problem of resolvents within a Hilbert space framework depends on a result of Greenstein [21] and leads to the independent interesting question of when is a generalized resolution of identity real analytic on an interval of the axis. The corresponding question involving absolute continuity rather than analyticity has been the subject of several recent investigations; one of the most recent being that of Schreiber [83]. In a form appropriate for the theory to be developed in this paper, Greenstein's result is as follows:

Theorem 1.1. A necessary and sufficient condition that an analytic function $f(\lambda)$ with positive real part, $\operatorname{Re} f(\lambda)>0$ for $\operatorname{Re} \lambda>0$, which in this region admits the representation

$$
f(\lambda)=\int_{-\infty}^{\infty} \frac{d m(\omega)}{i \omega-\lambda},
$$

admits an analytic continuation through an interval $a \leqq \operatorname{Im} \lambda \leqq b$ is that $m(\lambda)$ be real analytic for $\omega=\operatorname{Im} \lambda$ in this interval. When this condition is satisfied, the continuation to the region $\operatorname{Re} \lambda<0$ is given by the relation

$$
f(\lambda)=\overline{f(-\lambda)}-2 \pi m^{\prime}(-\lambda) .
$$

(Since the original formulation involved the upper half-plane, the necessary transformation for the above form is given in Appendix II for the reader's convenience.)

For the continuation problem, the main result to be developed in this paper is that of theorem 4.14 which implies that the matrix elements formed by the inner product of the resolvent of a maximal dissipative closed operator with functions of compact support are precisely functions admitting the representation necessary for the application of Greenstein's theorem. The continuation problem is therefore reduced to the question of discovering additional conditions sufficient to guarantee that the weight function involving the generalized resolution of the identity is real analytic. Unfortunately, this problem continues to remain open.

The other results to be developed here, however, are of intrinsic interest since as previously stated they represent a considerable generalization of the theory of so-called positive real functions as suggested by the correspondence between the theory of Laplace transform and the theory of semigroups. To initiate this correspondence, consider the system of differentia] equation 


$$
\frac{d p}{d t}-A p=f
$$

in which $\boldsymbol{p}$ and $\boldsymbol{f}$ are $n$ dimensional vectors in $R^{n}$ and $A$ an $n \times n$ constant matrix. A system of this form is general enough to encompass the equations of linear lumped passive networks. If it is solved by the method of Laplace transforms under the assumption (for convenience only) that all initial conditions are zero, then one finds if, for example,

$$
\hat{\boldsymbol{P}}=\int_{0}^{\infty} e^{-\lambda t} \boldsymbol{p} d t, \quad \operatorname{Re} \lambda>0,
$$

that

$$
\hat{\boldsymbol{P}}=(\lambda I-A)^{-1} F .
$$

This relationship, as in the theory of Fourès and Segal [16] is of the form, the transformed output is equal to the product of an admittance operator - the resolvent - or "gain" function and the transformed input. How if one uses the formal analogy between Laplace transform theory and semi-groups and, as in Hille and Phillips [29] page 337, one finds that the resolvent of the infinitesimal generator is the Laplace transform of the semi-group operator and conversely that the semi-group operator is obtainable from the resolvent by inversion of the Laplace integral for a suitable integration path $c$. If $S(t)$ denotes the semi-group operator and $R_{\lambda}(A)$ the resolvent of the generating operator $A$, then

$$
\begin{aligned}
\int_{0}^{\infty} e^{-\lambda t} S(t) d t & =R_{\lambda}(A), \\
\frac{1}{2 \pi i} \int_{c} e^{-\lambda t} R_{\lambda}(A) d \lambda & =S(t) .
\end{aligned}
$$

Thus one sees easily that the resolvent $(\lambda I-A)^{-1}$ clearly plays the role of an admittance operator whenever $A$ can be interpreted as the infinitesimal generator of a semi-group. Actually, although it is somewhat aside from our main considerations, one can use an observation of my colleague, W. Root, to the effect that "feedback systems» so fundamental to our automated society can also be interpreted as the theory of the resolvent. It is even possible to phrase much of the classical mathematical procedures in this language. For example, using this remark, I observed that any wellset elliptical problem of the form

$$
L \varphi=-\frac{\varphi}{\lambda}+f
$$


can be so interpreted by constructing the Green's function of

$$
L \varphi=0 .
$$

If the associated integral operator is denoted by $A$ again, the solution to the given problem is of the form

$$
\varphi=(\lambda I+A)^{-1}(\lambda A f)
$$

in which the right-hand side is in the canonical form of the feedback circuit shown in the figure below. Obviously, in this interpretation, the values of $\lambda$ for which $(\lambda I+A)^{-1}$ does not exist - the discrete spectra - must correspond to regimes of instability. While it will not be pursued here, the suggestion of $W$. Root that the entire known theory of feedback systems be re-examined from the point of view of resolvents and semi-groups is most suggestive and worthy of further investigation.

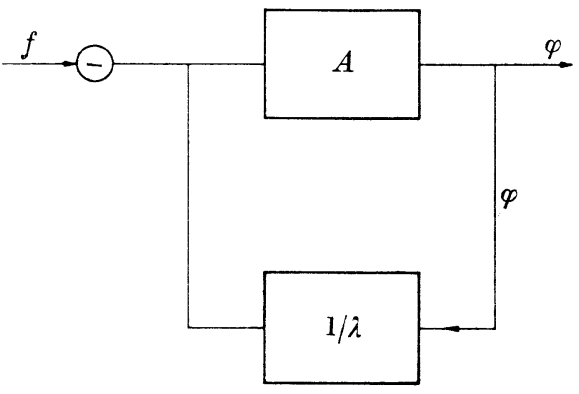

Fig. 1

Before pursuing in more detail the correspondence suggested by the above consideration, $\S 2$ will be devoted to a brief sketch of the occurrence of the "black box» or admittance (or impedance) concepts in physics and may be omitted either by physicists or by mathematicians who are not interested in possible applications. § 3 will summarize the properties of linear passive systems as they occur, for example, in network theory. $\S 4$ will be mainly devoted to a summary of the aspects of the theory of maximal dissipative closed operators necessary for the ensuing discussion. $\S 5$ will discuss some of the less obvious correspondences which exist between linear passive systems as treated in $\S 3$ and the theory developed in $\S 4$. In particular, the relationship between passivity and causality will be discussed in Hilbert space for regularly dissipative operators in the sense of Kato [39]. The paper concludes with two appendices, the first devoted 
to an example of Griffin in which the original method of Dolph and Penzlin [10] is used to construct an explicit representation of the resolvent of a dissipative operator and the second given over to the reformulation of Greenstein's theorem [21] appropriate for the right half-plane.

\section{The admittance or black box concept in physies}

In most physical theories two formulations are usually possible. The first and most historical approach is based on the concept of causality. The physical quantity, whether a voltage, a quantum wave function, an electromagnetic wave, etc. is assumed known at some definite time (the initial condition) and then calculated at a later time by means of an appropriate equation as, for example, Schrödinger's for the quantum wave problem. The method of calculation is in essence the method of semi-groups which often are time dependent (cf. the discussion in Kato [36], [38]). In the other formulation, a description of part of the state of the system - e.g. all incoming waves - is assumed to be known for all times and the remaining part of the state of the system - e.g. all outgoing waves - are then calculated for all times by means of a postulated interaction model. The interaction mechanism takes place in a region - the »black box» - about which usually little is known. In network theory where this formulation was first developed systematically, the interaction mechanism is usually represented by an admittance or impedance function which characterizes the frequency response properties of the network. The network in turn, if it is causal, can be realized in a number of equivalent ways, all of which have the same frequency response. In nuclear physics, the black box "corresponds to the so-called internal region" where the colliding particles are close together and interact strongly. Because of the nuclear scale, all real observations must take place essentially at infinity and thus it is natural that this second formulation would be more convenient for the discussion of asymptotic properties. The complicated internal mechanism is replaced by a boundary condition on the surface of the internal region and the theory involves only the asymptotic properties of the input and output states of the system, and the sadmittance» relation between them.

The usefulness of the »black box» point of view has been commented on by many authors including Heisenberg [26], [27], Wigner [100], Gross [22], Meixner [60], Zadeh [112], and Falkoff [14]. Although the last named author never published a paper corresponding to the following abstract, his summary is most succinct and will be quoted: 


\section{"Impedance Concept in Nuclear Scattering»}

"The description of the scattering of incident particles by complex nuclei has much in common with the methods used in describing the scattering of electromagnetic waves by obstacles or the effects of discontinuities in wave guide and transmission line problems. Many of the general features of both kinds of scattering, e.g. reciprocity conditions, conservation laws, resonant frequencies and connected formulas may be established within the framework of a common formalism. In particular, the familiar impedance concept of electrical network theory has its counterpart in the 'logarithmic derivative' of the wave function for the quantum mechanical description of the nuclear scattering. Hence all the well-known properties of the impedance as a function of frequency have immediate application to the energy dependence of nuclear cross sections. As a further example of the parallelism, the counterpart of the Breit-Wigner resonance formula and the Campbell-Foster theorem will be given.»

In the theories of the compound nucleus, these observations have been used extensively in the $R$-matrix theory of Wigner-Eisenbud [105] (cf. the other references to Wigner and the review article of Lane and Thomas [46]), as well as in the Kapur-Peierls [33] formalism (cf. also Peierls [65] and [67] and the review article by Brown [6]). Bloch [4] has presented a beautiful unification of these two approaches by introducing a Hamiltonian containing a singular boundary value operator. Upon its inversion the Wigner-Eisenbud or Kapur-Peierls theory results by a choice of an appropriate basis in the Hilbert space of the internal region. Finally, Siegert [87] introduced a theory based on radio-active states which is also closely related to the discussion by Petzold [68]. This theory was later extended by Humblet [31] by the use of the theory of meromorphic functions and as such is closely related to the method used by Nussenzveig [63].

There are other recent developments in physics which are also closely related to the matter at hand. For example, Regge in [73] and [74] applied the well-known (since 1918) Watson transformation (cf. Sommerfeld [88]), a frequently used device in more classical propagation problems, to continue the orbital momentum $j$ of the radial Schrödinger equation from discrete integral values to the complex plane. As a result he was able to show that under reasonable conditions, satisfied by most field theoretical potentials, the scattering amplitude at some fixed frequency determined the potential uniquely whenever it existed. Moreover, for the special class of time independent potentials which were capable of analytic continuation into a bounded region in the complex plane, the scattering amplitude 
can be continued to arbitrary large negative values of the cosine of the scattering angle and thus for arbitrary large real and positive transmitted momentum. This enables him to establish the Mandelstam dispersion relations over a wider range and has led to a better understanding of socalled shadow states, bound states, and resonances. Many physicists believe that the successful extensions of his ideas to quantum field theory hold much promise for the future (cf. Chew [8]).

The attempts at a quantum field theory adequate for unstable particles also involve ideas and methods which, if successful and rigorous, would require the existence of a transient theory of continuous systems. As of 1958, of the sixteen known elementary particles all but four were known to be unstable. Recents attempts at a theory of the unstable particles are based on a remark of Peierls [66] who suggested that the complex poles of the so-called "propagator» or resolvent in the second sheet of the Riemann surface can be used to account for the unstable states in the Lee model. This problem can also be viewed as a perturbation problem for a Hamiltonian with a continuous spectrum having a discrete eigenvalue embedded in it and the unstable states arise from analytic continuation through the continuous spectrum (cf. Zumino [113] and [114] and Lévy [53] and [54]). This subject is also intimately related to the work of Friedrichs [19], [20], Rejto [75], and Schwartz [84], who have develope da rigorous theory for sufficiently weak and smooth perturbations. On the other hand, as Lévy has remarked [53]:

"The great advantage of the propagators' method, apart from the fact that it is 'natural' and independent of the various production mechanisms, is that it involves only the assumption of a 'field' $\varphi$ but no asymptotic 'free' states into which the particles go when $t \rightarrow \infty$....'

"Unfortunately, the propagators' method has also a disadvantage: the Riemann surface in a realistic theory will have an infinite number of sheets, a new one starting at each value of the energy corresponding to the threshold of a new real process. Each of these sheets will contain poles, and an additional physical principle is necessary to decide which one has the correct physical meaning in order to represent the complete mass of an unstable particle.»

All of these theories, whether for networks, compound nucleus, or unstable particles, make extensive use of the concept of the resolvent (i. e. the admittance, the propagator, or the Green's function) and the methods of analytic continuation. The resolvent is, of course, at the heart of linear applied mathematics since once it has been constructed, usually in the appropriate concrete form of an impedance or admittance function 
or as an integral operator having a Green's function for its kernel, both the applied mathematician and physicist uses its singularities to obtain various expansion theorems and completeness relations. In mathematics it can be used as a basis for developing the spectral theory for both bounded and unbounded operators in a Hilbert spaces as in Stone [89] or Achieser and Glasman [1] and the projection properties associated with its contour integral over isolated parts of the spectrum not only form the basis for the rigorous perturbation theories of Sz.-Nagy [90] - [92] and Kato [34], [35] but represent the basis for an operational calculus of operators in Banach spaces (ef. e. g., Riesz and Sz.-Nagy [78], Taylor [95], [96], and Dunford [11]-[13]). Since until very recently, mathematicians concentrated on self-adjoint problems where the resolvent exists everywhere in the plane except on the real line (or imaginary axis in semi-group theory), the theory of analytic continuation has not been systematically combined with the theory of the resolvent, although as noted in [9], the continuation of the resolvent from one half-plane to another when possible, usually gives rise to new phenomenon discussed in $\S 1$. To repeat, it is hoped that the discussion here will stimulate and draw the attention of other mathematicians to this gap and will cause a new discipline to emerge.

\section{Linear passive systems and positive real functions}

A linear passive system is universally required to satisfy four conditions. If $I$ denotes a suitably chosen class of inputs and $O$ a corresponding class of outputs and if $L$ is used to denote the functional operation which assigns to each element $f$ of $I$ a unique element $f_{0}$ of $O$, the map $L f=f_{0}$ is required to be:

1. Linear; i.e. $L\left(a_{1} f_{1}+a_{2} f_{2}\right)=a_{1} L f_{1}+a_{2} L f_{2}$ when $a_{1}$, a are complex numbers;

2. Translational invariant in time; i.e. if $f, g \in I$ and $g(t)=L f(t)$ for all $t$ then $L f(t-s)=g(t-s)$ for all $t$;

3. Causal; i.e. if $f \in I$ is such that $f(t)=0$ for $t \leqq t_{0}$ then $L f(t)=0$ for $t \leqq t_{0}$;

4. Passive or dissipative; i.e. for any $f \in I$ with associated $L f \in O$, the condition

$$
\operatorname{Re} \int_{-\infty} L f * f d t \geqq 0 \quad \text { for all } t \leqq t_{0}
$$

must hold for a suitably defined product »*». 
The closely related concept of a linear scattering system is due to Meixner [60] and is sometime more convenient for some physical problems. In such a system with corresponding classes $I$ and $O_{1}$ postulates 1,2 and 3 are assumed to hold and postulate 4 is replaced by

4'. Passivity for scattering systems: i.e. for any $f \in I$ and associated $L f \in O_{1}$, the relation

$$
\operatorname{Re} \int_{-\infty}^{t} f(t) * f(t) d t \geqq \operatorname{Re} \int_{-\infty}^{t} L f * L f d t \quad \text { for all } t \leqq t_{0}
$$

must hold for a suitably defined product »*».

Now as Meixner observed, given the product "** there is a one-to-one correspondence between linear passive systems and linear scattering systems. This results from the fact that the condition of $4^{\prime}$ can be written as

$$
\operatorname{Re} \int_{-\infty}^{t}[f(t)-L f(t)] *[f(t)+L f(t)] d t \geqq 0
$$

so that if $f, L f$ are elements of a linear scattering system, then $f-L f$, $f+L f$ are elements in a linear passive system and vice versa.

According to Meixner [60] and Gross [22] the first three postulates are due to Duhamel and were also employed by Boltzman. The fourth postulate has only evolved comparatively recently although an incorrect form with the upper limit of the integral set equal to plus infinity has been used for some time in network theory (cf. Wu [109], Carlin [7], Oono [64], and Raisbeck [72]). The correct form was deduced by Meixner [58] from thermodynamic considerations and Youla, Castriota and Carlin [111] not only pointed out (by a counter example) that the plus infinity form may not be meaningful for even the simplest networks but they were the first to obtain the important result that postulate 4 - passivity - implies postulate 3 - causality. Their argument can be easily extended to the Hilbert space situation and this will be done in $\S 5$.

Depending on the choices made for the classes $I, O$ and the product "*» it is possible to derive slightly different theories. Thus to put continuity properties in the foreground and avoid the complications of generalized integrals and distribution theory, König and Meixner [44] chose $I$ to be the class of real functions $m$-times differentiable on the interval $-\infty<t<\infty$ (not necessarily the same interval for each function) and chose $O$ to be the corresponding class $C_{1}$ with "*» an ordinary product of two functions. Youla et al [111] chose $I$ and $O$ to be the Lebesque 
space of square-integral n-component column and row vectors with an inner product defined by

$$
(a, b)=\int_{-\infty}^{\infty} \bar{a}^{T} b d t
$$

where the bar denotes conjugation and $a^{T}$ the transpose of the column vector $a$. Wu [109] made a similar choice while, as already noted in the introduction, Fourès and Segal [16] chose $I$ and $O$ to be the pre-Hilbert spaces of the linear set of square integrable functions say $H$ on a real vector group $G$ to a separable complex Hilbert space $K$ with an inner product defined by

$$
(f, g)_{H}=\int_{G}(f(a), g(a))_{K} d a
$$

where the subscripts $» H »$ and $» K »$ indicate inner products in the respective space and $d a$ is the element of Lebesgue measure on $G$. Clearly their framework includes that of Youla et al [111] and $\mathrm{Wu}$ [109]. Here a similar situation will be used in $\S 5$ except attention will be restricted to the real line and a Bochner integral used.

In terms of these theories by introduction of a Laplace or Fourier transform it is possible to establish a relation of the form

$$
F_{0}(\lambda)=Y(\lambda) F(\lambda)
$$

where the $F, F_{0}$ are the transforms of $f, f_{0}$ and $Y$ represents the admittance or "gain» or resolvent. Restricting attention for the moment to the network case consisting either of lumped reactances (inductances and capacities) or relaxation elements (resistance and inductance or resistances and capacities) the admittance $Y\left(\lambda_{0}\right)$ is a so-called positive real function and has the following properties:

(1) It is analytic in the right-half plane except possibly on the imaginary axis;

(2) $\operatorname{Re} Y(\lambda)>0$ for $\operatorname{Re} \lambda>0$;

(3) Its real part in the first quadrant, with the exception of the imaginary axis must be positive;

(4) If it corresponds to a reactance network, it may have only simple poles on the imaginary axis;

(5) If it corresponds to a relaxation network, it may have poles only on the negative real axis and the residues at finite poles are negative real for $R L$ networks and positive real for $R C$ networks. 
It may be proved that a positive-real function admits the unique representation

$$
Y(\lambda)=A \lambda+\frac{B}{\lambda}+\int_{-\infty}^{\infty} \frac{1-i \omega \lambda}{\lambda-i \omega} d \varrho(\omega), \quad \operatorname{Re} \lambda \geqq 0,
$$

where

(a) $\varrho(\omega)$ is monotonically increasing, odd and bounded,

(b) $\varrho(\omega)=\frac{1}{2}[\varrho(\omega+0)+\varrho(\omega-0)]$,

(c) $\varrho(+0)-\varrho(-0)=0$.

In general $\varrho(\omega)$, the distribution function, is the sum of a discontinuous part $\varrho_{d}$, which is everywhere constant apart from its utmost denumerable points of discontinuity, a singular part $\varrho_{s}$, which is continuous but has a derivative $=0$ almost everywhere, and an absolutely continuous part $\varrho_{a}$, having a derivative almost everywhere. Electrical networks of the reactance type have distribution functions of the type $\varrho_{d}$, while other networks of lumped elements have spectral functions which in general consist of sums of $\varrho_{d}$ and $\varrho_{a}$. Examples have been constructed however, where special spectral functions of the singular type occur (cf. Wu and Pickard [110]).

While such a complete characterization of RLC networks of lumped constants cannot be given, their admittance are still regular in the right half-plane and they can possess only simple poles on the imaginary axis with positive real residues. Other poles may have any relative position and multiplicity. For all of these networks, however, a knowledge of the real part of the admittance or its reciprocal - the impedance - for $\lambda=i \omega$ completely characterizes $Y(\lambda)$ throughout the entire complex plane in the sense that one is able to construct $Y(\lambda)$ from a knowledge of the values of $\operatorname{Re} Y(\lambda)$ alone by use of, for example, the Stieltjes inversion formula. A detailed derivation of the properties given above can be found in the books by Bode [5], and Guillemin [23], [24].

One of the most important common results of the theories of Meixner and König, Youla et al, and $\mathrm{Wu}$ is that there is a one-to-one correspondence between the linear passive systems they consider and the class of real positive functions. Thus each linear passive system gives rise to a real positive function and conversely to each real positive function one can construct a linear passive system having the same real positive function for its admittance. The analogous result for regular dissipative operators and the more general linear passive system to which they correspond will be established in $\S 5$.

To conclude this section it should be mentioned that many but not all of the above properties persist if distributed inductances, capacities and 
resistances are permitted and some even for wave-guide circuits where the network equations have to be replaced by those of Maxwell. The interested reader is referred to the papers by Richards [76], [77], Wigner [101]-[103], and Fränz [17] where these matters are discussed in detail. It will be shown that the important properties of analyticity in a half-plane and rate of growth and representation all have their counterparts for maximal closed dissipative operators.

\section{Closed maximal dissipative and conservative operators}

The derivation of the relations which exist between the class of closed maximal dissipative operators in a Hilbert space and an appropriately defined linear passive system will be carried out in two steps. In this paragraph attention will be focused on the properties of maximal dissipative operators alone and the discussion will contain both a summary of published results and the proof of the new results which are needed. The following paragraph will relate these results to linear passive systems and discuss the relation between passivity and causality. The basic definition below and the theorems $4.1-4.10$ are all due to Phillips [70] although the first was obtained independently by Dolph and Penzlin [10].

Definition 4.1. Let $H_{0}$ be a Hilbert space with an inner product $(f, g)$. $A$ linear operator $A$ with domain $D(A)$ is said to be dissipative if

$$
2 \operatorname{Re}(A f, f)=(A f, f)+(f, A f) \leqq 0, \quad f \in D(A),
$$

and to be maximal dissipative if it is not a proper restriction of any other dissipative operator.

Theorem 4.1. Let $A$ be a dissipative operator and suppose $\lambda>0$. Then for $g=\lambda f-A f$ and $f$ in $D(A)$ one has $\|f\| \leqq\|g\|$, or for $R_{\lambda}(A) g=(\lambda I-A)^{-1} g=f$ one has $\left\|R_{\lambda}(A)\right\| \leqq 1 / \lambda$. Further the map $[f, A f] \rightarrow g$ of the graph $G(A)=\left\{f, A f ; f \in D(A) \subset H_{0} \times H_{0}\right\}$ cnto the range $R(I-A)$ is one-to-one and bicontinuous.

Theorem 4.2. Let $\lambda<0$ and suppose that $A$ is a dissipative operator with dense domain. Then $A$ is maximal dissipative if and only if the range $R(\lambda I-A)=H_{0}$.

Theorem 4.3. If $A$ is maximal dissipative and closed, then it has a dense domain.

Theorem 4.4. If $A$ is a maximal dissipative operator with dense domain, then so is its adjoint $A^{*}$.

Theorem 4.5. Let $A_{0}$ be a dissipative operator with dense domain and set $B_{1}=A_{0}^{*}$. Then there exist maximal dissipative operators $A$ and $B$, 2 
adjoints of each other and both having dense domains such that $A \supset A_{0}$ and $B \subset B_{1}$.

Definition 4.2. An operator $A$ is called conservative if

$$
(A f, f)+(f, A f)=0 \quad \text { for } \quad f \in D(A) \text {. }
$$

Theorem 4.6. The operator $A$ is conservative if and only if $i A$ is symmetric. If $A$ is conservative and maximal dissipative with dense domain the $i A$ is maximal symmetric. Conversely, if $i A$ is maximal symmetric with dense domain, then either $A$ or $(-A)$ is conservative and maximal dissipative. Finally $A$ and $A^{*}$ are conservative with dense domain if and only if $i A$ is self-adjoint.

Theorem 4.7. Suppose $A_{0}$ is a closed conservative operator with dense domain and set $A_{1}=-A_{0}^{*}$. Then each maximal dissipative extension of $A_{0}$ is a restriction of $A_{1}$.

Theorem 4.8. A necessary and sufficient condition for an operator $A$ to generate a strongly continuous semi-group of contraction operators on $H_{0}$ to itself is that $A$ be a maximal dissipative operator with dense domain.

Theorem 4.9. A necessary and sufficient condition for an operator $A$ to generate a strongly continuous semi-group of isometries is that $A$ be conservative and maximal dissipative with dense domain.

Theorem 4.10. A necessary and sufficient condition for a conservative maximal dissipative operator with dense domain to generate a strongly continuous group is that $i A$ be self-adjoint. In this case the group consists of unitary operators.

While the material will not be needed here for the sake of completeness, it should be mentioned that some of the above theorems have been generalized by Lumer and Phillips [56] to the semi-inner product Banach spaces introduced by Lumer [55].

It is well known in the theory of self-adjoint operators in Hilbert space that the resolution of the identity of the resolvent of a self-adjoint operator $A$ is the resolution of the identity of the operator $A$ and conversely (cf. [1] page 182). Partial results of this same sort are possible for maximal dissipative operators using the improved representation of the resolvent given in the next theorem following the suggestion of Phillips referred to in the introduction. Before stating this, recall the

Definition 4.3. A one parameter family of bounded self-adjoint operators $F(\omega)$ in the space $H_{0}$ is said to be a generalized resolution in the sense of Naimark [1] if

(a) $F\left(\omega_{1}\right)-F\left(\omega_{2}\right) \geqq 0$ if $\omega_{1}>\omega_{2}$,

(b) $F(-\infty)=O ; \quad F(+\infty)=I$ (the identity operator),

(c) $\quad F(\omega+0)=F(\omega)$. 
Naimark [1] also proved the following:

Theorem 4.11. If $F(\omega)$ is an arbitrary generalized resolution of the Hilbert space $H_{0}$, then there exists a larger Hilbert space $H$ which contains $H_{0}$ as a subspace and there exists an orthogonal resolution of the identity $E(\omega)$ such that for each $f \in H_{0}$ one has

$$
F(\omega) f=P E(\omega) f
$$

where $P$ is the projection operator from $H$ to $H_{0}$.

Since the proof of the new representation theorem depends essentially on theorems due to Sz.-Nagy and Stone, their precise statement is included for convenience.

Theorem 4.12. (Sz.-Nagy [93].) Let $\{S(t), t \geqq 0\}$ be a strongly continuous semi-group of contraction operators on $H_{0}$. Then there exists a group of unitary operators $\{U(t),-\infty<t<\infty\}$ on a larger Hilbert space $H$ containing $H_{0}$ as a subspace such that

$$
S(t) f=P U(t) P f \quad \text { for } \quad f \in H, \quad t \geqq 0 ;
$$

here $P$ is the projection operator with range $H_{0}$. The space $H$ can be constructed in a minimal fashion so that it is spanned by $U(t) f$ where $f$ is in $H_{0}$ and $-\infty<t<\infty$. In this case, the structure $\left\{H_{0}, U(t), H\right\}$ is determined to within an isomorphism.

Theorem 4.13. (Stone [89].) All weakly continuous groups of unitary transformations in a Hilbert space $H$ admit the spectral representation

$$
U(t)=\int_{-\infty}^{\infty} e^{i \omega t} d E(\omega)
$$

where $E(\omega)$ is a uniquely determined resolution of the identity.

It is now possible to state and prove the

Theorem 4.14. Let $A$ be a maximal dissipative and closed operator in the Hilbert space $H_{0}$. Then for any $\lambda$, Re $\lambda>0$, the resolvent exists and can be represented uniquely as

$$
R_{\lambda}(A)=(\lambda I-A)^{-1}=\int_{-\infty}^{\infty} \frac{d F(\omega)}{i \omega-\lambda}
$$

where $F(\omega)$ is a generalized resolution of the identity and hence the projection of orthogonal resolution of the identity in a space $H$ containing $H_{0}$ as a subspace. Further $H$ can be constructed in a minimal fashion, and

$2 \operatorname{Re}\left(R_{\lambda}(A) f, f\right)=\left(R_{\lambda}(A) f, f\right)+\left(f, R_{\dot{\lambda}}(A) f\right) \geqq 0 \quad$ for $\operatorname{Re} \lambda>0$. 
To establish this result, recall that by definition (Hille and Phillips [29]) that the resolvent of the semi-group $S(t)$ whose existence is guaranteed by theorem 4.8 is defined for any $f \in H_{0}$ by

$$
R_{\lambda}(A) f=(\lambda I-A)^{-1} f=\int_{0}^{\infty} e^{-i t} S(t) f d t .
$$

By Sz.-Nagy theorem it follows that

$$
R_{\lambda}(A) f=\int_{0}^{\infty} e^{-\lambda t} P U(t) f d t,
$$

and by Stone's theorem that this in turn is equal to

$$
R_{\lambda}(A) f=\int_{0}^{\infty} e^{-i t}\left\{\int_{-\infty}^{\infty} e^{i \omega t} P E(\omega) f\right\} d t,
$$

whence by Fubini's theorem

$$
\begin{aligned}
R_{\lambda}(A) f & =\int_{-\infty}^{\infty}\left\{\int_{0}^{\infty} e^{-i t+i \omega t} d t\right\} d(P E(\omega) f) \\
& =\int_{-\infty}^{\infty} \frac{d F(\omega) f}{i \omega-\lambda} .
\end{aligned}
$$

Since $R_{\lambda}(A)$ is a resolvent, it is defined for every $f$ in $\operatorname{Re} \lambda>0$ and hence has a range dense in $H_{0}$ and satisfies the Hilbert relations

$$
R_{\hat{\lambda}}(A)-R_{\mu}(A)=-(\lambda-\mu) R_{\dot{\lambda}}(A) R_{\mu}(A)
$$

thus the theorem is proved. Now by consideration of the adjoint as in [9] it follows that the representation is unique. Finally, if one sets

$$
\begin{gathered}
g=R_{\dot{\lambda}}(A) f, \\
(\lambda I-A) g=f,
\end{gathered}
$$

the inequality for dissipative operators implies that

$$
((\lambda I-A) g, g)+(g,(\lambda I-A) g) \geqq 0
$$

so that

$$
\left(R_{\hat{\lambda}}(A) f, f\right)+\left(f, R_{\hat{\lambda}}(A) f\right) \geqq 0 .
$$

In general the representation given here seems to involve a larger Hilbert space than that deduced from the original representation of Dolph 
and Penzlin [10]. It is clear from the method of proof here that $F(+\infty)=I$, a property not established for the family of monotone functions used in the representation given in [10]; and E. Griffin has found an example where the monotone family used there fails to reach the identity. However, when the monotone family, used in the original method of Dolph and Penzlin [10], is a generalized resolution of the identity then the extension can be carried out explicitly. An example of this is given in the appendix I. To the best of my knowledge, no one has as yet succeeded in carrying through a comparable example via the extension which depends on the theorem of Sz.-Nagy used above.

A precise converse to the above theorem is not known at this time. One can, however, prove the following:

Theorem 4.15. Let $F(\omega)$ be a generalized resolution of the identity and suppose:

(a) The one parameter bounded family of operators

$$
\varrho(\lambda)=\int_{-\infty}^{\infty} \frac{d F(\omega)}{i \omega-\lambda}
$$

exists and has a dense range for all $\lambda$ such that $\operatorname{Re} \lambda>0$.

(b) There exists one $\lambda_{0}$ with $\operatorname{Re} \lambda_{0}>0$ such that $\varrho\left(\lambda_{0}\right) f=0$ implies that $f=0$.

(c) The resolvent equations of Hilbert hold; namely,

$$
\varrho(\lambda)-\varrho(\mu)=-(\lambda-\mu) \varrho(\lambda) \varrho(\mu) \text {. }
$$

Then there exists a maximal dissipative closed operator $A$ whose resolvent is $\varrho(\lambda)$ for $\operatorname{Re} \lambda>0$ and moreover,

$$
2 \operatorname{Re}(\varrho(\lambda) f, f)=(\varrho(\lambda) f, f)+(f, \varrho(\lambda) f) \geqq 0 \quad \text { for } \operatorname{Re} \lambda>0 \text {. }
$$

To prove this, one first recalls that the hypotheses (b) and (c) are sufficient for theorem 4.10 of Stone [89] so that a unique closed linear operator $A$ will exist and have a resolvent coinciding with $\varrho(\lambda)$ for every $\lambda, \operatorname{Re} \lambda>0$. On the other hand, the representation (a) implies that

$$
\|\varrho(\lambda)\|<\frac{1}{\operatorname{Re} \lambda} \quad \text { for } \operatorname{Re} \lambda>0 .
$$

To obtain this estimate, the recent result of Schreiber's [83] will be used.

He proved that for any $\varphi$ in $L_{\infty}$

$$
\left|\int_{-\infty}^{+\infty} \varphi(\omega) d(F(\omega) f, g)\right| \leqq\|\varphi\|_{\infty} V[d(F(\omega) f, g)] \leqq\|\varphi\|_{\infty}\|f\|\|g\| .
$$


In this expression \|\|$_{\infty}$ is the $L_{\infty}$ norm and $V$ the total variation of the measure $d(F(\omega) f, g)$. Since

$$
\varphi=\frac{i}{i \omega-\lambda}
$$

in $(\varrho(\lambda) f, g)$ it follows that if $\lambda=x+i y$

$$
\|\varphi\|_{\infty}=\operatorname{ess} \sup \frac{1}{|i \omega-\lambda|}=\sup \frac{1}{\left[x^{2}+(\omega-y)^{2}\right]^{1 / 2}} \leqq \frac{1}{x} .
$$

so that choosing $g=\varrho(\lambda) f$ one has

$$
\|\varrho(\lambda) f\|^{2}=(\varrho(\lambda) f, \varrho(\lambda) f) \leqq \frac{\|f\|\|\varrho(\lambda) f\|}{\operatorname{Re} \lambda}
$$

from which the desired estimate follows.

Theorems by Foiaș [15] for Hilbert spaces and Kato [37] for locally sequentially weakly compact Banach spaces imply that the properties of dense range and the Hille-Yosida criterion are sufficient to guarantee that the operator $A$ defined by Stone's theorem has a dense domain. In turn, an operator satisfying the Hille--Yosida criterion and having a dense domain is the infinitesimal generator of a strongly continuous family of contraction operators. Finally, theorem 4.8 implies that the operator $A$ must be maximal dissipative as well as closed. This in turn, as in theorem 4.15 implies $\operatorname{Re} \varrho(\lambda)>0$. The main features of the last two results may be summarized in the

Theorem 4.16. A necessary and sufficient condition that a one-parameter family of bounded operators having the representation of theorem 4.15 be the resolvent of a maximal dissipative operator in the half-plane $\operatorname{Re} \lambda>0$ is that hypotheses of theorem 4.15 hold for them.

While the statement of this theorem is probably sufficient for most purposes, it would be of interest to have a necessary and sufficient condition stated in terms of the measure defined by $F(\omega)$ instead of the assumption in regard to the resolvent equations.

For the sake of completeness, the following two, as yet unpublished, theorems of Lebow [52] should be mentioned in this section, rephrased in the right half-plane of analyticity.

Theorem 4.17. If $A$ is a maximal dissipative closed operator, then proper vectors of real proper values of $i A$ are proper vectors of $(i A)^{*}$.

Theorem 4.18. If $A$ is a maximal dissipative closed operator, then $i A$ has no real residual spectrum.

To summarize briefly, this section has demonstrated that the resolvents of maximal dissipative operators possess properties of regularity and 
representation which are completely analogous to those of the so-called positive real functions. For this reason, they might be termed "positive real resolvents" and henceforth in this paper, they will be referred to in this manner.

Definition 4.4. A one-parameter family of bounded operators satisfying the hypotheses of theorem 4.15 will be called a positive real resolvent.

\section{Positive real resolvents and linear passive Hilbert systems}

If $A$ denotes the maximal dissipative closed operator associated with a positive real resolvent it is natural to associate with it the system

$$
\frac{d u}{d t}-A u=f(t)
$$

in which $u=u(t)$ and $f(t)$ are functions from the real line to a Hilbert space $H$ and to ask to what extent does this abstract equation of evolution possess properties analogous to those of linear passive systems.

Since $A$ is in general an unbounded operator, the hypothesis that $A$ is the infinitesimal generator of a strongly continuous semi-group of contraction operators is not sufficient to guarantee the existence of a solution to (5.1) in general (cf. Kato [36]). Several sufficient additional conditions are, however, known. Thus Phillips [69] proved that (5.1) has a strong solution in Banach space if $A$ satisfies the Hille-Yosida criterion in the weak form due to him (cf. [36]) provided that $f(t)$ was strongly continuous; Kato [36] has proved that a solution will exist in a Banach space also if the conditions on $f(t)$ are replaced by the assumption that $f(t)$ is in the domain of $A$ for every $t$ and if it is assumed that $A f(t)$ and $f(t)$ are strongly continuous in $t$.

For Hilbert spaces, the condition that $\operatorname{Re}(A u, u) \leqq-\alpha(u, u)$ hold for some $\alpha>0$ for $A$ maximal dissipative and closed is also sufficient to guarantee that the associated semi-group $S(t)$ will have an exponential decay so that the solution to (5.1) will be square integrable with $f(t)$.

This addition hypothesis is, of course, the usual required in the LaxMilgram lemma [48] if $A$ is replaced by $(-A)$.

A somewhat more general sufficient condition of this same type can be obtained by specializing some recent results of Kato [38], [39] obtained for equations of the form (5.1) when $A$ is allowed to depend on the time. Recall that a complex-valued function $\varphi(u, v)$ is called a sesquilinear form in a Hilbert space $H$ if for $u, v$ belonging to a linear subset $D$ the domain - contained in $H, \varphi$ is linear in $u$ and anti-linear in $v$. 
Also recall that if $\varphi$ is a sesquilinear form the relation $\varphi^{*}(u, v)=\overline{\varphi(v, u)}$ defines another sesquilinear form $\varphi^{*}$ - the adjoint form - with domain $D\left(\varphi^{*}\right)=D(\varphi)$. Finally, recall that any sesquilinear form $\varphi$ can be written as

$$
\varphi=\operatorname{Re} \varphi+\operatorname{Im} \varphi
$$

with

$$
\begin{aligned}
& \operatorname{Re} \varphi=\frac{1}{2}\left(\varphi+\varphi^{*}\right), \\
& \operatorname{Im} \varphi=\frac{1}{2}\left(\varphi-\varphi^{*}\right) .
\end{aligned}
$$

Kato [39] calls a sesquilinear form $\varphi$ regular if:

(a) $D(\varphi)$ is dense in $H$.

(b) $\operatorname{Re} \varphi$ is closed, $(\operatorname{Re} \varphi)^{*}=\operatorname{Re} \varphi$, and non-negative in the sense that there exists a constant $\alpha>0$ such that $\operatorname{Re} \varphi(u, u) \geqq \alpha(u, u)$.

(c) There exists a $\beta \geqq 0$ such that

$$
|\operatorname{Im} \varphi(u, u)| \leqq \beta \operatorname{Re} \varphi(u, u) .
$$

Kato then proved the following:

Theorem 5.1. Let $\varphi$ be a regular sesquilinear form. Then there is a unique closed, maximal dissipative operator $(-B)$ with domain $D(B) \subset D(\varphi)$ such that

$$
\varphi(u, v)=(B u, v) \quad \text { for } u \in D(B) \text { and } v \in D(\varphi) .
$$

$(-B)$ is called the regular dissipative operator associated with the regular sesquilinear form $\varphi$. Similarly $\left(-B^{*}\right)$ is the maximal dissipative operator associated with $\varphi^{*}$, where $\varphi(u, v)=\left(u, B^{*} v\right)$ for $u \in D(\varphi)$ and $v \in D\left(B^{*}\right)$ and $D\left(B^{*}\right) \subset D(\varphi)$.

Sufficient conditions for a closed maximal dissipative operator $(-B)$ to be regular dissipative are not known, but Kato [39] has shown that if the inequality (5.2) holds for $\beta<1$, then $(-B)$ is regularly dissipative.

Specializing theorems I and III of Kato [38], one can now conclude by identifying $(-B)$ with $A$ in equation (5.1) that the following is true:

Theorem 5.2. If $S(t)$ is the semi-group of strongly continuous contraction operators generated by the regular dissipative operator $A$, then if $f(t)$ is continuous in $t$, any strong solution of (5.1) must be expressible in the form

$$
u(t)=S(t-0) u(0)+\int_{0} S(t-s) f(s) d s .
$$

Conversely, the $u(t)$ given by (5.3) is a strict solution of (5.1) if $f(t)$ is Hölder continuous on closed interval $[0, T]$ for $u(0)$ an arbitrary element of $H$. 
Other sufficient conditions are also known, (cf. e.g., Kato and Tanabe [40]) and unquestionably somewhat weaker hypotheses will be discovered in the future. Since the relationship between positive real resolvents and linear systems of the form (5.1) fails to be exactly parallel to that between positive real functions and linear passive systems only because of the necessity for additional hypotheses sufficient to guarantee a solution to (5.1), an attempt will be made in so far as is now possible to phrase this relationship independent of any specific additional hypotheses.

To investigate this relationship, let $d t$ denote an element of measure on $R^{1}$ and recall the

Definition 5.1. A function $f: R^{1} \rightarrow H_{0}$ is measurable if and only if $(f(t), g)$ is Lebesgue measurable on $R^{1}$ for every $g$ in $H_{0}$.

Definition 5.2. A function $f: R^{1} \rightarrow H_{0}$ is said to be Bochner integrable if and only if it is measurable and

$$
\int_{R^{1}}\|f(t)\| d t<\infty
$$

The Bochner integral of $f$ is then defined by

$$
\left(\int_{R^{1}} f(t) d t, g\right)=\int_{R^{1}}(f(t), g) d t
$$

and satisfies

$$
\left\|\int_{R^{1}} f(t) d t\right\| \leqq \int_{R^{1}}\|f(t)\| d t
$$

Using the notation of Wilcox [107], recall the

Definition 5.3. The space

$$
L_{2}\left(R^{1} ; H_{0}\right)=\left\{f, f \text { measurable and } \int_{R^{1}}\|f\|^{2} d t<\infty\right\}
$$

is a Hilbert space with a scalar product

$$
[f, g]=\int_{R^{1}}(f(t), g(t)) d t
$$

In particular, $L_{2}\left(R^{1} ; R^{1}\right)$ is the usual space of Lebesgue square-integrable complexvalued functions on the real line $R^{1}$ and this is the space that occurs in the theory of Youla et al [111] and Wu [109]. 
Definition 5.4. The space

$$
L_{2}^{d}=L_{2}\left(\frac{d}{d t}, R^{1}, H_{0}\right)=\left\{f, f \in L_{2}\left(R^{1}, H_{0}\right) \text { and } \frac{d f}{d t} \in L_{2}\left(R^{1}, H_{0}\right)\right\}
$$

This is a Hilbert space with a scalar product defined by

$$
\langle f, g\rangle=\int\left\{(f(t), g(t))+\left(\frac{d f(t)}{d t}, \frac{d g(t)}{d t}\right)\right\} d t .
$$

The following theorem on integration by parts established by Wilcox [107] will also be needed:

Theorem 5.3. If $f, g$ are in $L_{2}^{d}$, then

$$
\int_{t_{1}}^{t_{2}}\left[\left(f^{\prime}(t), g(t)\right)+\left(f(t), g^{\prime}(t)\right)\right] d t=\left(f\left(t_{2}\right), g\left(t_{2}\right)\right)-\left(f\left(t_{1}\right), g\left(t_{1}\right)\right)
$$

for any $-\infty<t_{1}<t_{2}<\infty$.

Henceforth, attention will be restricted to functions either inputs or outputs which are in the space $L_{2}^{d}$ even though several results would still be true under somewhat weaker hypotheses. For example, to establish the fact that the passivity postulate stated below is sufficient to imply causality, one merely must assume that integrals of the form

$$
\int_{-\infty}^{s}\left[(f(t), f(t))+\left(f^{\prime}(t), f^{\prime}(t)\right)\right] d t<\infty
$$

exist for all $s,-\infty<s \leqq t_{0}$, for functions which have the property that

$$
\lim _{t \rightarrow-\infty}(f(t), f(t))=0 \text {. }
$$

As noted earlier, in the scalar case this is an important generalization since the above integral can fail to exist for important examples if $s=+\infty$. Here the situation is unclear at this time so that, for simplicity, the results will be stated for functions in $L_{2}^{d}$. In any case, should this restriction prove to limiting, it is a simple matter to check which arguments are still valid.

With this in mind, one has

Definition 5.5. A linear relation of the form

$$
L u=\frac{d u}{d t}-A u=f(t)
$$


in which $u=u(t)$ and $f(t)$ are functions from the real line to the Hilbert space $L_{2}^{d}$ and where $A$ is a time independent operator defined on $L_{2}$, will be said to be a linear passive Hilbert system if:

(1) The domain $D(L)=D(A) \cap L_{2}^{d}$ is dense in $L_{2}$.

(2) The equation $L u=f$ has a solution in $L_{2}^{d}$ for every $f$ in $L_{2}^{d}$ (i.e., the range of the operator $L$ contains $L_{2}^{d}$ ).

(3) The system is passive in the sense that the inequality

$$
\operatorname{Re} \int_{-\infty}^{s}(L(u, t), u(t)) d t \geqq 0
$$

is valid for all $u \in L_{2}^{d}$ and all $s, s \leqq t_{0}$.

Since the operator $A$ is independent of $t$, the above system will also be obviously invariant under translations in time as is an ordinary linear passive system.

Theorem 5.4. Any linear passive Hilbert system is causal in the sense that if $f \in L_{2}^{d}$ has the property that $f(t)=0$ for $-\infty \leqq t \leqq t_{0}$ and if $L u=f$ then $u(t)=0$ for all $t \leqq t_{0}$.

To prove this, let $L u_{1}=f_{1}$ and $L u_{2}=f_{2}$ for $u_{1}, u_{2}$ in $L_{2}^{d}$. By linearity and passivity one has

$$
\begin{aligned}
& 0 \leqq \operatorname{Re} \int_{-\infty}^{s}\left(\left(a_{1} f_{1}+a_{2} f_{2}\right),\left(a_{1} u_{1}+a_{2} u_{2}\right)\right) d t \\
& =\left|a_{1}\right|^{2} \operatorname{Re} \int_{-\infty}^{s}\left(f_{1}, u_{1}\right) d t+\left|a_{2}\right|^{2} \operatorname{Re} \int_{-\infty}^{s}\left(f_{2}, u_{2}\right) d t \\
& +\operatorname{Re}\left[a_{1} \bar{a}_{2} \int_{-\infty}^{s}\left(f_{1}, u_{2}\right) d t+\bar{a}_{1} a_{2} \int_{-\infty}^{s}\left(f_{2}, u_{1}\right) d t\right] \\
& =\left|a_{1}\right|^{2} \operatorname{Re}\left(f_{1}, u_{1}\right) d t+\left|a_{2}\right|^{2} \operatorname{Re}_{-\infty}^{s}\left(f_{2}, u_{2}\right) d t \\
& +\frac{1}{2} a_{1} \bar{a}_{2}\left\{\int_{-\infty}^{s}\left[\left(f_{1}, u_{2}\right)+\left(u_{1}, f_{2}\right)\right] d t\right\} \\
& \left.+\frac{1}{2} \bar{a}_{1} a_{2} \mid \int_{-\infty}^{s}\left[\left(f_{2}, u_{1}\right)+\left(u_{2}, f_{1}\right)\right] d t\right\} .
\end{aligned}
$$


Thus, as in the proof of Cauchy's inequality, it follows that the passivity inequality and linearity imply that

$$
\left|\int_{-\infty}^{s}\left[\left(f_{1}, u_{2}\right)+\left(f_{2}, u_{1}\right)\right] d t\right|^{2} \leqq 4 \operatorname{Re}\left[\int_{-\infty}^{s}\left(f_{1}, u_{1}\right) d t\right] \operatorname{Re}\left[\int_{-\infty}^{s}\left(f_{2}, u_{2}\right) d t\right] .
$$

Now suppose that $f_{1}(t)=0$ for $t \leqq t_{0}$. Then this inequality implies that

$$
\int_{-\infty}^{s}\left(f_{2}, u_{1}\right) d t=0, \quad s \leqq t_{0} \text {. }
$$

Choosing $f_{2}=u_{1}(t)$ in $t \leqq t_{0}$ implies that $u_{1}(s)=0$ almost everywhere for $s \leqq t_{0}$. This is moreover the only possible response to the input $f_{1}$. To verify this one computes and repeats the same argument with $u_{1}(t)$ equal to the difference of two assumed solutions corresponding to the same input so that $f_{1}(t)=0$.

Systems satisfying definition (5.6) include those of the following:

Theorem 5.5. The system

$$
\frac{d u}{d t}-A u=f(t)
$$

considered in the space $L_{2}^{d}$ is a linear passive Hilbert system if $A$ is regularly dissipative, and its resolvent is a positive real resolvent.

Since $A$ is regularly dissipative and $f$ is in $L_{2}^{d}$, a solution will exist by Kato's theorem [38], and since $A$ is regularly dissipative, it is maximal dissipative and closed and hence it will have a positive real resolvent by the results of $\S 4$. It only remains to verify that the passivity postulate holds. For this, it is sufficient to compute

$$
\begin{aligned}
& \left(\frac{d u}{d t}, u\right)-(A u, u)=(f, u), \\
& \left(u, \frac{d u}{d t}\right)-(u, A u)=(u, f),
\end{aligned}
$$

from which, by theorem 5.3, it follows that

$$
\begin{aligned}
(u(s), u(s)) & -\int_{-\infty}^{s}[(A u, u)+(u, A u)] d t \\
& =\int_{-\infty}^{s}\left[\left(\frac{d u}{d t}, u\right)+\left(u, \frac{d u}{d t}\right)\right] d t-\int_{-\infty}^{s}[(A u, u)+(u, A u)] d t \\
& =2 \operatorname{Re} \int_{-\infty}^{s}(f(t), u(t)) d t=2 \operatorname{Re} \int_{-\infty}^{s}(L(u), u) d t \geqq 0 .
\end{aligned}
$$


The last inequality follows from the fact that the first member of this chain or relation is positive in virtue of the fact that $A$, being regularly dissipative, is maximal dissipative and closed.

Conversely, one has the

Theorem 5.6. Sufficient conditions that a one-parameter family of bounded operators having a dense range and the representation

$$
\varrho(\lambda)=\int_{-\infty}^{\infty} \frac{d F(\omega)}{i \omega-\lambda}
$$

in the region $\operatorname{Re} \lambda>0$ be the resolvent of a linear passive Hilbert system are: (1) $\varrho(\lambda)$ be a positive real resolvent; (2) the operator $A$ whose resolvent is $\varrho(\lambda)$ be regularly dissipative.

This theorem is, of course, an immediate consequence of theorem 4.15 and the discussion of this section once the expression $d u / d t-A u=f(t)$ is formed.

To conclude, it is apparent from the discussion that it is not possible to state necessary and sufficient conditions at this time.

\section{Appendix I}

\section{An application of the Dolph-Penzlin theorem}

The following example was worked out by E. Griffin and represents a case where the procedure of Dolph and Penzlin [10] does lead to a generalized resolution of the identity. In particular, $F(\infty)=I$, and the example is interesting in that one obtains a representation of a two dimensional operator in terms of $L_{2}$ over the real line. To avoid confusion with the extension theorem 3.14, the operator will be chosen to have its resolvent regular in the upper half-plane. Under these circumstances the matrix

$$
A=\left(\begin{array}{rr}
-i & 1 \\
1 & 0
\end{array}\right)
$$

satisfies the condition for a dissipative operator in the sense that

$$
\frac{A-A^{*}}{2 i}=\operatorname{Im} A \leqq 0 \text {. }
$$

Setting $z=x+i y$ one finds directly

$$
R(z)=(A-z I)^{-1}=\frac{\left(\begin{array}{cc}
-z & -1 \\
-k & -(i+z)
\end{array}\right)}{D_{0}}
$$


where $D_{0}=z^{2}+i z-1$ has zeros in the lower-half $z$ plane at $(-i \pm \sqrt{3}) / 2$ so that $R(z)$ is seen to be analytic for $\operatorname{Re} z>0$ in accordance with the general theory. If $U=\left(\begin{array}{l}m \\ n\end{array}\right)$ then

$$
\begin{aligned}
(R(z) U, U) & =\frac{1}{D_{0}}\left(\left(\begin{array}{c}
-z m-n \\
-m-i n-z n
\end{array}\right),\left(\begin{array}{c}
m \\
n
\end{array}\right)\right) \\
& =\frac{-z\left[|m|^{2}+|n|^{2}\right]-(m \bar{n}+\bar{m} n)-i||^{2}}{D_{0}} .
\end{aligned}
$$

Using $\operatorname{Im}(a+b i) /(c+d i)=\left|\begin{array}{ll}c & d \\ a & b\end{array}\right| /\left(c^{2}+d^{2}\right)$, it follows that

$$
\operatorname{Im}(R(z) U, U)=\frac{\left|\begin{array}{lr}
\left(x-y^{2}-y-1\right) & (x+2 x y) \\
\left\{-x\left(|m|^{2}+|n|^{2}\right)-(m \bar{n}+\bar{m} n)\right\} & \left(-|n|^{2}\right)
\end{array}\right|}{D_{0}} .
$$

The Stieltjes inversions formula can be used to find the generalized resolution of the identity $F(\omega)$. Setting $y=0$ and proceeding heuristically, one finds, in differential form:

$$
\operatorname{Im}(R(x) U, U)=\frac{1}{|D|^{2}}\left|\begin{array}{l}
x^{2}-1 \\
\left\{-x\left(|m|^{2}+|n|^{2}\right)-(m \bar{n}+\bar{m} n)\right\}\left(-|n|^{2}\right)
\end{array}\right| .
$$

where $|D|^{2}=\left|D_{0}(x)\right|^{2}=\left(x^{2}-1\right)^{2}+x^{2}$.

Choosing base vectors $U_{1}=\left(\begin{array}{l}1 \\ 0\end{array}\right), \quad U_{2}=\left(\begin{array}{l}0 \\ 1\end{array}\right)$ and polarizing, one finds

$$
\begin{aligned}
& \operatorname{Im}\left(R(x) U_{1}, U_{1}\right)=\frac{1}{|D|^{2}}\left|\begin{array}{ll}
x^{2}-1 & x \\
-x & 0
\end{array}\right|=\frac{x^{2}}{D^{2}}, \\
& \operatorname{Im}\left(R(x) U_{1}, U_{2}\right)=\operatorname{Im}\left(R(x) U_{2}, U_{1}\right)=\frac{x}{|D|^{2}}, \\
& \operatorname{Im}\left(R(x) U_{2}, U_{2}\right)=\frac{1}{|D|^{2}} .
\end{aligned}
$$

Thus the matrix has the form

$$
\begin{aligned}
& \left(\operatorname{Im}\left(R(x) U_{i}, U_{j}\right)\right)=\left(\begin{array}{ll}
x^{2} /\left(\pi|D|^{2}\right) & x /\left(\pi|D|^{2}\right) \\
x /\left(\pi|D|^{2}\right) & 1 /\left(\pi|D|^{2}\right)
\end{array}\right) \\
& =\left(\begin{array}{l}
x /(\sqrt{\pi}|D|) \\
1 /(\sqrt{\pi}|D|)
\end{array}\right)(x /(\sqrt{\pi}|D|) \quad 1 /(\sqrt{\pi}|D|)) .
\end{aligned}
$$


The functions $x /(\sqrt{\pi}|D|)$ and $1 /(\sqrt{\pi}|D|)$ are orthogonal in $L_{2}(-\infty, \infty)$. The orthogonality follows at once since

$$
\left(\frac{x}{|D|}, \frac{1}{|D|}\right)=\int_{-\infty}^{\infty} \frac{x}{|D|^{2}} d x=0,
$$

since one is integrating an odd function over an even integral. To show that they are properly normalized, consider for example

$$
\begin{aligned}
\int_{-\infty}^{\infty} \frac{1}{\pi|D|^{2}} d t & =\frac{1}{\pi} \int_{-\infty}^{\infty} \frac{d t}{t^{4}-t^{2}+1} \\
& =\frac{1}{\pi} \int_{-\infty}^{\infty} \frac{d t}{\left(t-e^{i \pi / 6}\right)\left(t-e^{-i \tau / 6}\right)\left(t+e^{i \pi / 6}\right)\left(t+e^{-i \tau / 6}\right)} .
\end{aligned}
$$

This contour can be closed in the lower half-plane and evaluated by the calculus of residues to the obvious poles so that one obtains

$$
\begin{aligned}
\int_{-\infty}^{\infty} \frac{1}{\pi|D|^{2}} d t & -2 i \\
= & \frac{-2 i}{2\left(e^{-i \pi / 6}-e^{i \pi / 6}\right)\left(e^{-i \pi / 6}+e^{i \pi / 6}\right) e^{-i \pi / 6}} \\
& +-2\left(-e^{i \pi / 6}-e^{-i \pi / 6}\right)\left(-e^{i \pi / 6}+e^{-i \pi / 6}\right) e^{i \pi / 6} \\
= & \frac{e^{i \pi / 6}+e^{-i \pi / 6}}{\sqrt{3}}=1 .
\end{aligned}
$$

A similar calculation shows that

$$
\int_{-\infty}^{\infty} \frac{t^{2}}{\pi|D|^{2}} d t=1
$$

It is now possible to inbed $R(z)$ in $L_{2}(-\infty, \infty)$ by mapping

$$
U_{1} \rightarrow \frac{x}{\sqrt{\pi}|D|}, \quad U_{2} \rightarrow \frac{1}{\sqrt{\pi}|D|} .
$$

If one defines $M$ to be the subspace spanned by the above function, it is also easy to see that for any $f$ in $M$

$$
(R(z) f, f)=\int_{-\infty}^{\infty} \frac{|f(t)|^{2} d t}{t-z}
$$


For this it suffices to compute $\left(R(z) U_{i}, U_{j}\right)$. Thus, for example,

$$
\begin{aligned}
\left(R(z) U_{1}, U_{1}\right) & =\int_{-\infty}^{\infty} \frac{t^{2} d t}{\pi|D|^{2}(t-z)} \\
& =\frac{e^{i \pi / 6} e^{-i \pi / 3}}{\sqrt{3}\left(e^{-i \pi / 6}-z\right)}+\frac{e^{-i \pi / 6} e^{i \pi / 3}}{\sqrt{3}\left(-e^{i \pi / 6}-z\right)}=-\frac{z}{D_{0}} .
\end{aligned}
$$

Similarly,

$$
\begin{gathered}
\left(R(z) U_{1}, U_{2}\right)=\left(R(z) U_{2}, U_{1}\right)=\int_{-\infty}^{\infty} \frac{t d t}{\pi|D|^{2}(t-z)}=-\frac{1}{D_{0}}, \\
\left(R(z) U_{2}, U_{2}\right)=\int_{-\infty}^{\infty} \frac{d t}{\pi|D|^{2}(t-z)}=-\frac{i+z}{D_{0}} .
\end{gathered}
$$

Thus one recovers the original resolvent from this embedding. Moreover, it follows that the operator $F(t)$ represents the projection of the interval $(-\infty, \infty)$ onto $(-\infty, t)$ and that extended operator of the matrix $A$, say $A^{+}$is simply multiplication by $t$.

\section{Appendix II}

\section{A theorem of Greenstein for the right half-plane}

In [21], Greenstein proved:

Theorem A.II. If $g(z)$ is given by

$$
g(z)=\int_{-\infty}^{\infty} \frac{d \gamma(t)}{t-z}, \quad \operatorname{Im} g(z)>0 \quad \text { for } \operatorname{Im} z>0,
$$

where $\gamma(t)$ is a mass distribution normalized by

$$
\gamma(-\infty)=0, \quad \gamma(t-0)=\gamma(t), \quad-\infty<t<\infty,
$$

then $g(z)$ can be continued across $(a, b)$ into the lower half-plane if and only if $\gamma(t)$ is real analytic in $(a, b)$. If $\gamma(t)$ is real analytic in $(a, b)$ then the continuation of $g(z)$ across $(a, b)$ is given by

$$
g(z)=\overline{g(\bar{z})}+2 \pi i \gamma^{\prime}(z)
$$

To translate this result to the right-half plane, given $f(z)$ a function such that $\operatorname{Re} f(z)>0$ for $\operatorname{Re} z>0$, choose

$$
g(z)=i f(-i z), \quad s=i t, \quad m(s)=\gamma(-i s)
$$


the representation for $g(z)$ implies that

$$
i f(-i z)=i \int_{-i \infty}^{i \infty} \frac{d m(s)}{s-i z}
$$

or, if $\lambda=-i z, s=i u, u$ real, that

$$
f(\lambda)=\int_{-\infty}^{\infty} \frac{d m(u)}{u-\lambda}, \quad \operatorname{Re} \lambda>0 .
$$

Assuming continuation possible, it follows that

$$
i m^{\prime}(-\lambda)=\gamma^{\prime}(z) \quad \text { for } \operatorname{Im} z<0 \text { and } \operatorname{Re} \lambda<0,
$$

so that the above continuation formula becomes

$$
f(\lambda)=-\overline{f(-\bar{\lambda})}-2 \pi m^{\prime}(-\lambda) \quad \text { for } \quad \operatorname{Re} \lambda<0 .
$$

Note added in proof-reference (25 May 1963)

To page 6: A preprint by A. Grossman entitled "Nested Hilbert spaces in quantum mechanics. In is now available from the Institute for Advanced Study, Princeton (N.J.). The author's abstract is as follows: "A nested Hilbert space is a pair of Hilbert spaces $H_{0}, H_{1}$, each of which is in a certain sense identified with a dense subset of the other." (As in the Friedrichs' extension of semi-bounded operators; C. L. D.) "These structures are used here to study analytic continuation into 'unphysical sheets' and to discuss non-normalizable states of quantum-mechanical system."

\section{Acknowledgments}

The author wishes to express his thanks for the continuing interest and comments of his friend, Professor J. Meixner. He is also indebted to his colleagues Professors Allen Shields, E. H. Rothe, H. Schaeffer and to Professors Arlen Brown of Rice, Tosio Kato of Berkeley, Arnold Lebow of New York University, and M. Schreiber of the Courant Institute for their interest and constructive comments.

University of Michigan

Ann Arbor, Michigan, U.S.A. 


\section{References}

[1] Achieser, N. I., and I. M. Glasman: Theorie der linearen Operatoren im Hilbert-Raum. - Mathematische Lehrbücher 4, Akademie-Verlag, Berlin, 1954.

[2] Beck, G., and H. M. Nussenzveig: On the physical interpretation of complex poles of the $S$-Matrix. I. - Nuovo Cimento (10) 16, 1960, pp. 416-449.

[3] Beurling, A.: On two problems concerning linear transformations in Hilbert space. - Acta Math. 81, 1949, pp. 239-255.

[4] Bloch, C.: Une formulation unifiée de la théorie des réactions nucléaires. Nuclear Phys. 4, 1957, pp. 503-528.

[5] Bode, H. W.: Network analysis and feedback amplifier design. - Bell Telephone Laboratories Series, D. Van Nostrand Company, New York/Toronto/ London, 1945.

[6] Brows, G. E.: Foundations of the optical model for nuclei and direct interaction. - Rev. Mod. Phys. 31, 1959, pp. 893-919.

[7] CARLIN, H. J.: The scattering matrix in network theory. - IRE Trans. CT-3, 1956 , pp. $88-96$.

[8] Chew, G. F.: S-matrix theory of strong interactions without elementary particles. - University of California, Lawrence Radiation Laboratory UCRL-10058, 1962.

[9] DoLph, C. L.: Recent developments in some non-self-adjoint problems of mathematical physics. - Bull. Amer. Math. Soc. 67, 1961, pp. 1-69.

[10] Dolph, C. L., and F. Penzlin: On the theory of a class of non-self-adjoint operators and its applications to quantum scattering theory. - Ann. Acad. Scient. Fennicæ A. I. 263, 1959.

[11] Duxford, N.: Spectral theory. I. Convergence to projections. - Trans. Amer. Math. Soc. 54, 1943, pp. 185-217.

[12] - - Spectral theory. II. Resolutions of the identity. - Pacific J. Math. 2, 1952, pp. $559-614$.

[13] - - Spectral operators. - Pacific J. Math. 4, 1954, pp. 321-354.

[14] Falkoff, D. L.: Impedance concept in nuclear scattering. - Phys. Rev. 93, 1954, p. 364.

[15] Foiaș, C.: La mesure harmonique-spectrale et la théorie spectrale des opérateurs généraux d'un espace de Hilbert. - Bull. Soc. Math. France 85, 1957, pp. $263-282$.

[16] Fourès, Y., and I. E. Segal: Causality and analyticity. - Trans. Amer. Math. Soc. 78,1955, pp. $385-405$.

[17] Fränz, K.: Das Reaktanztheorem für beliebige Hohlräume. - Elektr. Nachr. Techn. 21, 1944, pp. 8-12.

[18] Friedman, B.: Propagation in a non-homogeneous medium. - Electromagnetic waves, Mathematics Research Center Publication 6, University of Wisconsin Press, Madison (Wisc.), 1962, pp. 301-310. 
[19] Friedrichs, K. O.: On the perturbation of continuous spectra. - Comm. Pure Appl. Math. 1, 1948, pp. 361-406.

[20] - - Perturbation of spectra in Hilbert space. - Lectures in Applied Mathematics 3, American Mathematical Society, Providence (R. I.), 1963.

[21] Greenstein, D. S.: On the analytic continuation of functions which map the upper half plane into itself. - J. Math. Anal. Appl. 1, 1960, pp. 355-362.

[22] Gross, B.: Lineare Systeme. - Nuovo Cimento (10) 3 Supplemento, 1956, pp. $235-296$.

[23] Guillemin, E. A.: The mathematics of circuit analysis. - Technology Press Book, Massachusetts Institute of Technology, John Wiley \& Sons/ Chapman \& Hall, NewYork/London, 1949.

[24] - - Synthesis of passive networks. - John Wiley \& Sons, NewYork/London, 1957.

[25] Halmos, P. R.: Shifts on Hilbert spaces. - J. Reine Angew. Math. 208, 1961, pp. $102-112$.

[26] Heisenberk, W.: Die »beobachtbaren Grössen» in der Theorie der Elementarteilchen. - Z. Physik 120, 1943, pp. 513-538.

[27] - - Die beobachtbaren Grössen in der Theorie der Elementarteilchen. II. Z. Physik 120, 1943, pp. 673-702.

[28] Heitler, W.: The quantum theory of radiation. - 3rd edition, International Series of Monographs in Physies, Clarendon Press, Oxford, 1954.

[29] Hille, E., and R. S. Phillips: Functional analysis and semi-groups. - 2nd revised edition of the book by E. HrLle under the same title, Colloquium Publications XXXI, American Mathematical Society, Providence (R. I.), 1957.

[30] HöHLeR, J.: Über die Exponentialnäherung beim Teilchenzerfall. - Z. Physik 152,1958 , pp. $546-565$.

[31] Humblet, J.: Sur la définition des niveaux virtuelles des noyaux atomiques et l'établissement de la formule de dispersion. - Mém. Soc. Roy. Sci. Liège (4) 12:4, 1952 .

[32] Ikebe, T.: Eigenfunction expansions associated with the Schroedinger operators and their applications to scattering theory. - Arch. Rational Mech. Anal. 5, 1960, pp. 1-34.

[33] Kapur, P. L., and R. Peierls: The dispersion formula for nuclear reactions. Proc. Roy. Soc. London A. 166, 1938, pp. 277-295.

[34] Kato, T.: On the convergence of the perturbation method. I. - Progr. Theoret. Phys. 4, 1949, pp. 514-523.

[35] - 》- On the convergence of the perturbation method. II. - Progr. Theoret. Phys. 5, 1950, pp. 207-212.

[36] - - On linear differential equations in Banach spaces. - Comm. Pure Appl. Math. 9, 1956, pp. 479-486.

[37] - - Remarks on pseudo-resolvents and infinitesimal generators of semigroups. - Proc. Japan Acad. 35, 1959, pp. 467-468.

[38] - - Abstract evolution equations of parabolic type in Banach and Hilbert spaces. - Nagoya Math. J. 19, 1961, pp. 93-125.

[39] -»- Fractional powers of dissipative operators. - J. Math. Soc. Japan 13, 1961, pp. $246-274$.

[40] Kato, T., and H. TanaBe: On the abstract evolution equation. - Osaka Math. J. 14, 1962, pp. 107-133. 
[41] Kralfin, L. A.: Contribution to the decay theory of a quasi-stationary state. Soviet Physics JETP 6 (33), 1958, pp. 1053-1063.

[42] KönIG, H.: Zur Theorie der linearen dissipativen Transformationen. - Arch. Math. 10, 1959, pp. 447-451.

[43] -"- Lineare dissipative Systeme. - Colloque sur l'analyse fonctionelle à Louvain 1960, Centre Belge de Recherches Mathématiques, Librairie Universitaire / Librairie Gauthier-Villars, Louvain / Paris, 1961, pp. $71-80$.

[44] König, H., and J. Meixner: Lineare Systeme und lineare Transformationen - Math. Nachr. 19, 1958, pp. 265-322.

[45] ЛАдыженская, О. А.: О принципе предельной амплитуды. - Успехи Мат. Наук $12: 3,1957$, pp. $161-164$.

[46] Lane, A. M., and R. G. Thomas: $R$-matrix theory of nuclear reactions. Rev. Mod. Phys. 30, 1958, pp. 257-353.

[47] LAX, P. D.: Translation invariant spaces. - Acta Math. 101, 1959, pp. 163-178.

[48] Lax, P. D., and A. N. Milgram: Parabolic equations. - Contributions to the theory of partial differential equations, Annals of Mathematics Studies 33, Princeton University Press, Princeton (N. J.), 1954, pp. 167-190.

[49] Lax, P. D., Cathleen S. Morawetz and R. S. Phillips: The exponential decay of solutions of the wave equation in the exterior of a star-shaped obstacle. - Bull. Amer. Math. Soc. 68, 1962, pp. 593-595.

[50] Lax, P. D., and R. S. Phillifs: The wave equation in exterior domains. - Bull. Amer. Math. Soc. 68, 1962, pp. 47-49.

[51] - - - - Spectral theory for the wave equation. - To appear in Comm. Pure Appl. Math.

[52] Leвow, A.: On von Neumann's theory of spectral sets. - To appear in J. Math. Anal. Appl.

[53] LÉvy, M.: On the description of unstable particles in quantum field theory. Nuovo Cimento (10) 13, 1959, pp. 115-143.

[54] - - Description of unstable particles in quantum field theory. - Lectures on field theory and the many-body problem, Academic Press, NewYork/ London, 1961, pp. 47-66.

[55] Lumer, G.: Semi-inner-product spaces. - Trans. Amer. Math. Soc. 100, 1961, pp. $29-43$.

[56] Lumer, G., and R. S. Phillips: Dissipative operators in a Banach space. Pacific J. Math. 11, 1961, pp. 679-698.

[57] McKelvey, R.: The spectra of minimal self-adjoint extensions of a symmetric operator. - Pacific J. Math. 12, 1962, pp. 1003-1022.

[58] Meixner, J.: Thermodynamische Erweiterung der Nachwirkungstheorie. Z. Physik 139, 1954, pp. 30-43.

[59] -»- Reversibilität und Irreversibilität in linearen passiven Systemen. Z. Naturf. 16 a, 1961, pp. $721-726$.

[60] - - The theory of linear passive systems. - Lecture notes, University of London, 1961.

[61] - - Relaxionerscheinungen und ihre thermodynamische Behandlung. Nederl. Tijdschr. Natuurk. 26, 1962, pp. 259-273.

[62] Nussenzveig, H. M.: The poles of the $S$-matrix of a rectangular potential well or barrier. - Nuclear Phys. 11, 1959, pp. 499-521.

[63] - - On the physical interpretation of complex poles of the $S$-matrix. II. Nuovo Cimento (10) 20, 1961, pp. 694-714. 
[64] Oono, Y.: Application of scattering matrices to synthesis of $n$-poles. - IRE Trans. CT-3, 1956, pp. 111-120.

[65] Peierls, R. E.: Expansions in terms of sets of functions with complex eigenvalues. - Proc. Cambridge Philos. Soc. 44, 1948, pp. 242-250.

[66] -»- Interpretation and properties of propagators. - The proceedings of the 1954 Glasgow conference on nuclear \& meson physics, Pergamon Press, London/NewYork, 1955, pp. 296-299.

[67] - - - Complex eigenvalues in scattering theory. - Proc. Roy. Soc. London A. 253,1959 , pp. $16-36$.

[68] Petzold, J.: Wie gut gilt das Exponentialgesetz beim $\alpha$-Zerfall? - Z. Physik 155,1959 , pp. $422-432$.

[69] Phillips, R. S.: Perturbation theory for semi-groups of linear operators. Trans. Amer. Math. Soc. 74, 1953, pp. 199-221.

[70] - - Dissipative operators and hyperbolic systems of partial differential equations. - Trans. Amer. Math. Soc. 90, 1959, pp. 193-254.

[71] - - Semigroup methods in the theory of partial differential equations. Modern mathematics for the engineer II, University of California, Engineering Extension Series, McGraw-Hill Book Company, New York/ Toronto/London, 1961, pp. 100-132.

[72] Raisbeck, G.: A definition of passive linear networks in terms of time and energy. - J. Appl. Phys. 25, 1954, pp. 1510-1514.

[73] RegGe, T.: Introduction to complex orbital momenta. - Nuovo Cimento (10) 14, 1959, pp. $951-976$.

[74] - - Bound states, shadow states and Mandelstam representation. - Nuovo Cimento (10) 18, 1960, pp. 947-956.

[75] Rejto, P. A.: On gentle perturbations. - New York University, Courant Institute, U. S. Army Research Office, DA-ARO-D 31, 174, 6159, 1962.

[76] Richards, P. I.: A special class of functions with positive real part in a halfplane. - Duke Math. J. 14, 1947, pp. 777-786.

[77] - - General impedance-function theory. - Quart. Appl. Math. 6, 1948, pp. $21-29$.

[78] Riesz, F., and B. Sz.-NaGy: Functional analysis. - Frederick Ungar Publishing Co., New York, 1955.

[79] Sснӧnberg, M.: Sur la théorie des perturbations en mécanique quantique. I. Spectres discontinus. - Nuovo Cimento (9) 8, 1951, pp. 242-270.

[80] - - Sur la théorie des perturbations en mécanique quantique. II. Spectres continus et mixtes. - Nuovo Cimento (9) 8, 1951, pp. $403-431$.

[81] " Physical applications of the resolvent operators. I. On the mathematical formalism of Feynman's theory of the positron. - Nuovo Cimento (9) 8 , 1951 , pp. $651-682$.

[82] - - - On the general theory of damping in quantum mechanics. - Nuovo Cimento (9) 8, 1951, pp. $817-842$.

[83] Schreiber, M.: Absolutely continuous operators. - Duke Math. J. 29, 1962, pp. $175-190$.

[84] Schwartz, J.: Mass renormalization and spectral shifts. - New York University, Courant Institute, NONR 285-40, ONR-USN, 1960.

[85] Schwinger, J.: Field theory of unstable particles. - Ann. Physics 9, 1960. pp. $169-193$.

[86] Serdobol'skir, V. I.: On the theory of scattering via quasi-stationary states. - Soviet Physics JETP 9 (36), 1959, pp. 1354-1357. 
[87] Siegert, A. J. F.: On the derivation of the dispersion formula for nuclear reactions. - Phys. Rev. 56, 1939, pp. 750-752.

[88] Sommerfeld, A.: Partial differential equations in physics. - Pure and Applied Mathematics I, Academic Press, New York, 1949.

[89] Sтоnе, M. H.: Linear transormations in Hilbert space and their applications to analysis. - Colloquium Publications XV, American Mathematical Society, New York, 1932.

[90] DE Sz. NAGY, B.: Perturbations des transormations autoadjointes dans l'espace de Hilbert. - Comment. Math. Helv. 19, 1946, pp. 347-366.

[91] -»- On uniformly bounded linear transformations in Hilbert space. - Acta Sci. Math. Szeged 11, 1947, pp. 152-157.

[92] Sz.-NAGY, B.: Perturbations des transformations linéaires fermées. - Acta Sci. Math. Szeged 14, 1951, pp. 125-137.

[93] - - Extensions of linear transformations in Hilbert space which extend beyond this space. - Appendix to [78], Frederick Ungar Publishing Co., New York, 1960.

[94] Sz.-NAGY, B., and C. Foias: Sur les contractions de l'espace de Hilbert. VI. Calcul fonctionnel. - Acta Sci. Math. Szeged 23, 1962, pp. 130-167.

[95] Taylor, A. E.: Analysis in complex Banach spaces. - Bull. Amer. Math. Soc. 49,1943 , pp. $652-669$.

[96] -»- Spectral theory of closed distributive operators. - Acta Math. 84, 1951, pp. $189-224$.

[97] Titchmarsh, E. C.: Some theorems on perturbation theory. III. - Proc. Roy. Soc. London A. 207, 1951, pp. 321-328.

[98] - - - Some eigenfunction expansions formulae. - Proc. London Math. Soc. (3) 11,1961, pp. $159-168$.

[99] - - A problem in relativistic quantum mechanics. - Proc. London Math. Soc. (3) 11,1961 , pp. 169-192.

[100] Wigner, E. P.: Nuclear reactions and level widths. - Amer. J. Phys. 17, 1949, pp. $99-109$.

[101] - - - On a class of analytic functions from the quantum theory of collisions. - Ann. of Math. (2) 53, 1951, pp. 36-67.

[102] - - On the connection between the distribution of poles and residues for an $R$ function and its invariant derivative. - Ann. of Math. (2) 55, 1952, pp. $7-18$.

[103] - - Simplified derivation of the properties of elementary transcendentals. Amer. Math. Monthly 59, 1952, pp. 669-683.

[104] - - On the development of the compound nucleus model. - Amer. J. Phys. 23, 1955, pp. 371-380.

[105] Wigner, E. P., and L. Eisenbud: Higher angular momenta and long range interaction in resonance reactions. - Phys. Rev. 72, 1947, pp. 29-41.

[106] Wigner, E. P., and J. v. Neumann: Significance of Loewner's theorem in the quantum theory of collisions. - Ann. of Math. (2) 59, 1954, pp. 418-433.

[107] Wilcox, C. H.: The mathematical foundations of diffraction theory. - Electromagnetic waves, Mathematics Research Center Publication 6, University of Wisconsin Press, Madison (Wisc.), 1962, pp. 65-98.

[108] Wu, T. T.: Causality and the radiation condition. - Harvard University, Cruft Laboratory Report 211, 1954.

[109] - - Causality and the frequency response function. - Harvard University, Cruft Laboratory Report 223, 1955. 
[110] WU, T. T., and W. F. Pickard: A numerical study of an unfamiliar type of impedance. - Harvard University, Cruft Laboratory Report 300, 1959.

[111] Youla, D. C., L. J. Castriota and H. J. Carlin: Bounded real scattering matrices and the foundations of linear passive network theory. - IRE Trans. CT-6, 1959, pp. $102-124$.

[112] ZADEH, L. A.: A general theory of linear signal transmission systems. - J. Franklin Inst. 253, 1952, pp. 293-312.

[113] Zumino, B.: On the formal theory of collision and reaction processes. - New York University, Courant Institute Report CX-23, 1956.

[114] -»- Unstable particles and complex poles of the propagators. - Lectures on field theory and the many-body problem, Academic Press, NewYork/ London, 1961, pp. $37-45$. 